\title{
Form, Symmetry and Asymmetry of the Dental Arch: Orthogonal Analysis Revisited
}

\author{
Toby Hughes, Lindsay Richards, and Grant Townsend \\ Dental School, The University of Adelaide, South Australia, 5005.
}

ABSTRACT There have been numerous attempts to quantify the shape of the dental arch mathematically, with orthogonal polynomial curves providing a robust and versatile method for quantifying variation in both shape and asymmetry. Lu (1966) first presented the theoretical basis for fitting orthogonal polynomials to

The shape of the dental arches has held the attention of physical anthroplogists and dentists since the beginning of the last century. Many methods have been developed to describe dental arch morphology, ranging from simple geometric classifications (Hrdlička, 1916), through combinations of linear dimensions (Moorrees, 1959) to various complex curve-fitting procedures (Lu, 1966; Jones and Richmond, 1989; Kasai et al., 1995; Battagel, 1996). The application of fourth-order polynomials of the form:

$$
y=a+b x+c x^{2}+d x^{3}+e x^{4}
$$

provides a number of advantages, the most significant being that the coefficients can be easily interpreted (Richards et al., 1990). The second ( $x^{2}$ or quadratic) and fourth ( $x^{4}$ or quartic) terms describe the arch shape while the first ( $x^{1}$ or linear) and third ( $x^{3}$ or cubic) terms describe asymmetry.

$\mathrm{Lu}$ (1966) drew attention to the inter-dependence of coefficients of simple polynomials. The sum of squares associated with the $k$ coefficients cannot be partitioned into $k$ parts, each attributable to a single degree of freedom. Consequently, it is not possible to assign accurate values to the relative contributions of symmetry and asymmetry to overall arch shape. The partition can be achieved, however, by using orthogonal polynomials (Kendall, 1959). Lu (1966) presented the first detailed account of fitting orthogonal polynomials to arch data. Unfortunately, although the theory for equally-spaced $\mathrm{x}$-coordinates was sound,

Editor's Note: Lu's paper (1966) has been cited many times in the dental literature, but researchers have been confused by the nature of the analysis (orthogonal regression is not the same as conventional regression analysis) and most biologists and clinicians have been unable to "break-through" Lu's mathematics, particularly since there are several key errors in the paper. Toby Hughes and his colleagues were invited to submit this paper to facilitate the understanding and application of this useful analytic method. arch shape data. Whilst theoretically sound, Lu's original paper contained several arithmetic errors and a number of incorrect assumptions. In this paper we present corrections for these errors and extrapolate the theory to unequally-spaced arch shape data using a simple recursive procedure first developed by Robson (1959).

Lu's worked example contained some mathematical errors. Furthermore, the extrapolation to non-equally spaced data was flawed. Kendall (1959) provided the correct general parameterization for unequally-spaced data, and this was further simplified to a recursive method by Robson (1959). The aim of the current paper is to address the errors within Lu's orginal paper, and to present a valid extrapolation of his work to unequallyspaced arch data for use in quantitative assessments of arch form.

\section{RESULTS AND DISCUSSION}

The theoretical workings presented on pages 10581062 of Lu's original paper are substantially correct, with one small exception, and it would be inappropriate to reproduce this section in great detail. We urge those readers who are interested in Lu's adaptation of orthogonal theory that produces a partition of arch shape variance for equally-spaced data to examine the original paper, noting that the calculation for the sums of squares for the intercept of the orthogonal regression on page 1059 reads:

$$
\operatorname{SS}\left(b_{0} \xi_{0}\right)=\frac{\sum \mathrm{Y}^{2}}{\mathrm{n}}
$$

when it should instead read:

$$
\operatorname{SS}\left(b_{0} \xi_{0}\right)=\frac{\left(\sum \mathrm{Y}\right)^{2}}{\mathrm{n}}
$$

Theoretically, Lu's concept cannot be faulted. However, the application of the theory was flawed, particularly in the use of published orthogonal polynomial tables for equally-spaced data (Fisher and Yates, 1957), leading to biased estimates of orthogonal coefficients. In the next section of this paper we reproduce verbatim the worked example from Lu's original paper

Correspondence to: Toby Hughes, Level 6 Dental School, University of Adelaide University, Australia 5005

E-mail: toby.hughes@adelaide.edu.au 


\section{AN ILLUSTRATIVE EXAMPLE}

Although the curve-fitting technique is well known in statistical circles, it is thought that the intended audience of this paper might not be as familiar, and for this reason the sample is explained in considerable detail.

The arch width is divided into 14 equidistant intervals defined by 15 points. We have the following observed data:

$X$ (arch base): -7, -6, -5, -4, -3, -2, -1, 0, 1, 2, 3, 4, 5, 6, 7

$Y$ (arch height): 27.7, 20.1, 13.9, 9.4, 6.1, 3.4, 1.4, 0, 0.3, $1.3,3.2,6.3,11.0,18.2,29.0$

The computations are illustrated in Table 1.

$$
\begin{array}{ll}
\sum Y \xi_{1}=\mathbf{4 3 . 2} \mathbf{c}^{\mathrm{c}} & \sum \xi_{1}{ }^{2}=280 \\
\sum Y \xi_{2}=\mathbf{6 9 3 2 . 2 ^ { \mathrm { a } }} & \sum \xi_{2}{ }^{2}=\mathbf{3 7 , 1 2 8 ^ { \mathrm { a } }} \\
\sum Y \xi_{3}=\mathbf{- 6 8 4 . 4 ^ { \mathrm { a } }} & \sum \xi_{3}{ }^{2}=\mathbf{3 9 , 7 8 0 ^ { \mathrm { a } }} \\
\sum Y \xi_{4}=\mathbf{- 1 0 5 , 9 5 8 . 6} & \sum \xi_{4}{ }^{\mathrm{ab}}=\mathbf{6 , 4 6 6 , 4 6 0} \mathbf{a b}
\end{array}
$$

\section{Explanatory notes}

From observed data, we compute $\sum Y^{2}$ and $\sum Y$.

2. Column 1 is obtained by adding the $Y$ values pairwised from the centre, e.g. $1.4+0.3=1.7 ; 3.4+1.3$ $=4.7$, etc. ${ }^{\mathrm{c}}$

3. Column 2 is obtained by subtracting the $Y$ value corresponding to $X$ from the $Y$ value corresponding to $-X$, e.g. $1.4-0.3=1.1 ; 3.4-1.3=$ 2.1, etc. ${ }^{c}$

4. Columns 3, 4, 5 and 6 are obtained from the orthogonal polynomial tables ${ }^{\mathrm{a}}$ (Fisher and Yates, 1957) with $\mathrm{n}=15$. The $\sum \xi_{i}^{2}$ are also obtainable from this table. These values are only listed for the upper half of the entire polynomial. ${ }^{\mathrm{c}}$ For evenpowered $\xi$ the omitted half are duplicates of the exhibited half; for odd-powered $\xi$ the omitted half are numerically the same values as the exhibited half, except with the signs reversed.

5. To obtain $\sum Y \xi_{1}$ and $\sum Y \xi_{3}$ we obtain the sum of cross products of the differences (Column 2) with Column 3 and Column 5 respectively. ${ }^{\mathbf{c}}$

6. To obtain $\sum Y \xi_{2}$ and $\sum \xi_{4^{\prime}}$ we obtain the sum of cross products of the sum (Column 1) with Column 4 and Column 6, respectively. ${ }^{c}$

$$
\begin{aligned}
& \text { are as follows: } \\
& \sum Y \xi_{1}=-43.2 \\
& \sum \xi_{1}{ }^{2}=280 \\
& \sum Y \xi_{2}=2,310.7 \\
& \sum \xi_{2}{ }^{2}=4,125 \\
& \sum Y \xi_{3}=821.3 \\
& \sum \xi_{3}{ }^{2}=57,283 \\
& \sum Y \xi_{4}=2,590.2 \\
& \sum \xi_{4}{ }^{2}=760,139
\end{aligned}
$$

Due to the calculational errors noted above plus a number of subsequent arithmetic and typographical errors, the remainder of the worked example was substantially incorrect. The correct parameterization with the associated partition of variation (Table 2) is presented below:

$$
\begin{array}{ll}
\sum Y^{2}=2848.55 & \sum Y=151.3 \\
\sum Y \xi_{1}=-43.2 & \sum Y \xi_{2}=2,310.7 \\
\sum Y \xi_{3}=821.3 & \sum Y \xi_{4}=2,590.2
\end{array}
$$


TABLE 1. Computational table for fitting a fourth order orthodongal polynomial to 15 points

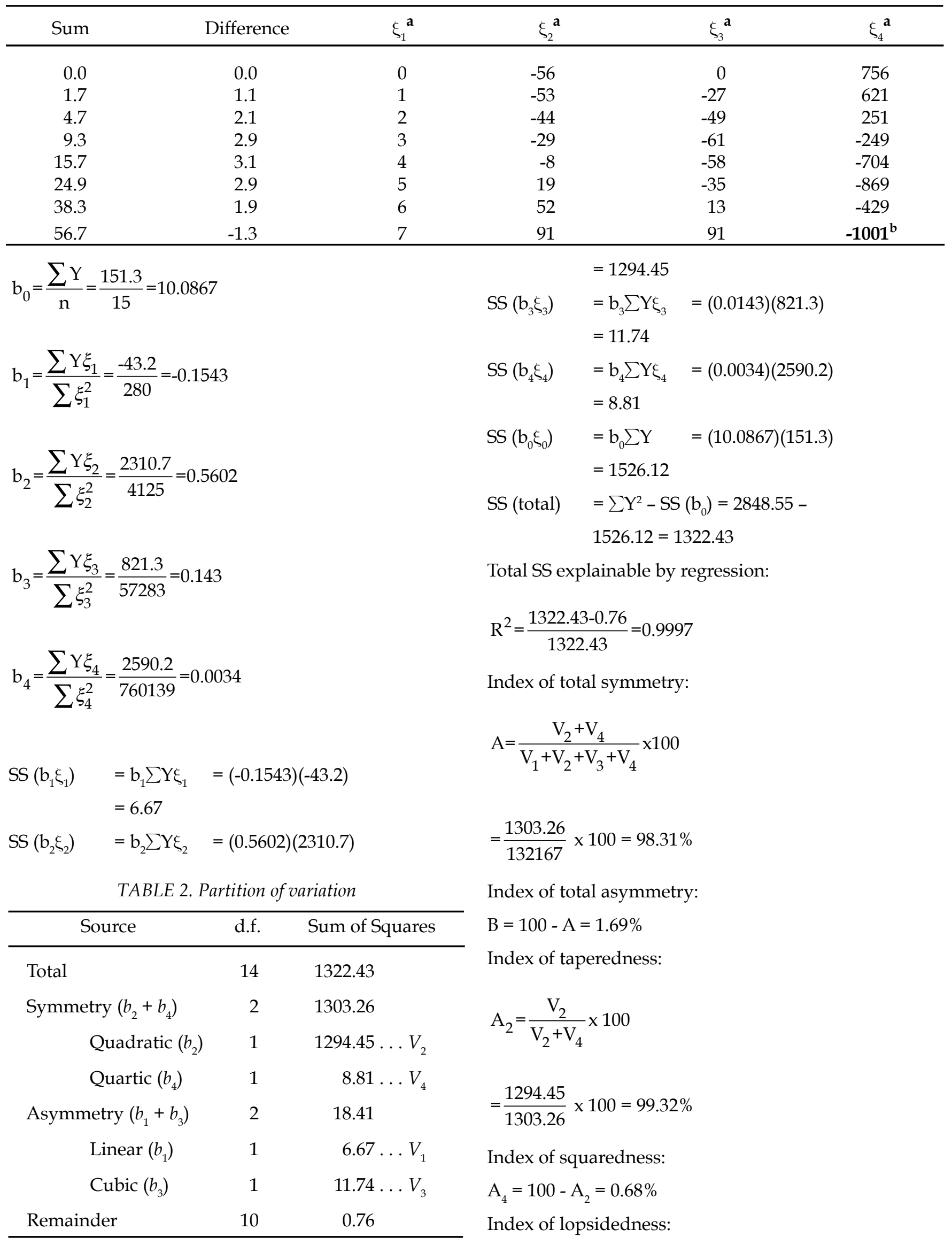




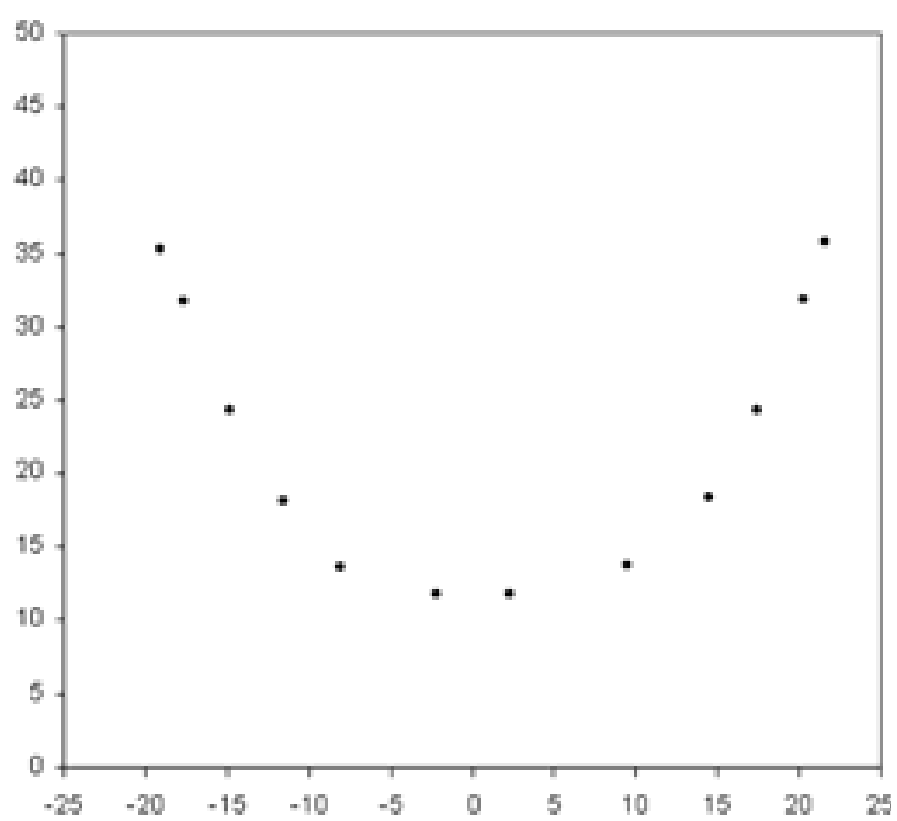

Fig. 1. Graphical representation of cusp tip spacings used to define arch shape.

$\mathrm{B}_{1}=\frac{\mathrm{V}_{1}}{\mathrm{~V}_{1}+\mathrm{V}_{3}} \times 100=\frac{6.67}{18.41}=36.26 \%$

Index of tiltedness:

$\mathrm{B}_{3}=100-\mathrm{B}_{1}=63.77 \%$

The procedure outlined above is suitable for data obtained at equidistant increments of $X$. However, on most occasions, investigators wish to define the dental arch in terms of specific anatomical landmarks. In such cases, the width distances of the arch may increase unequally and the use of tabulated orthogonal coefficients is invalid. Lu's (1966) analytical extension to unequally-spaced data was flawed, irrespective of the numerous typographical errors that were present in the derivation. Lu noted that in computing the following simple polynomial regressions:

$\mathrm{Y}=\mathrm{a}+\mathrm{b}_{1} \mathrm{x}$

$Y=a^{\prime}+b^{\prime}{ }^{\prime} x+b_{2} x^{2}$

$Y=a^{\prime \prime}+b^{\prime \prime}{ }_{1} x+b_{2}^{\prime} x^{2}+b_{3} x^{3}$

$\mathrm{Y}=\mathrm{a}^{\prime \prime \prime}+\mathrm{b}^{\prime \prime \prime}{ }_{1} \mathrm{x}+\mathrm{b}_{2}{ }_{2} \mathrm{x}^{2}+\mathrm{b}_{3} \mathrm{x}^{3}+\mathrm{b}_{4} \mathrm{x}^{4}$

it can be shown that:

$$
\mathrm{Y}=\mathrm{a}-\mathrm{b}_{1} \varphi+\mathrm{b}_{2} \varphi^{2}+\mathrm{b}_{3} \varphi^{3}+\mathrm{b}_{4} \varphi^{4}
$$

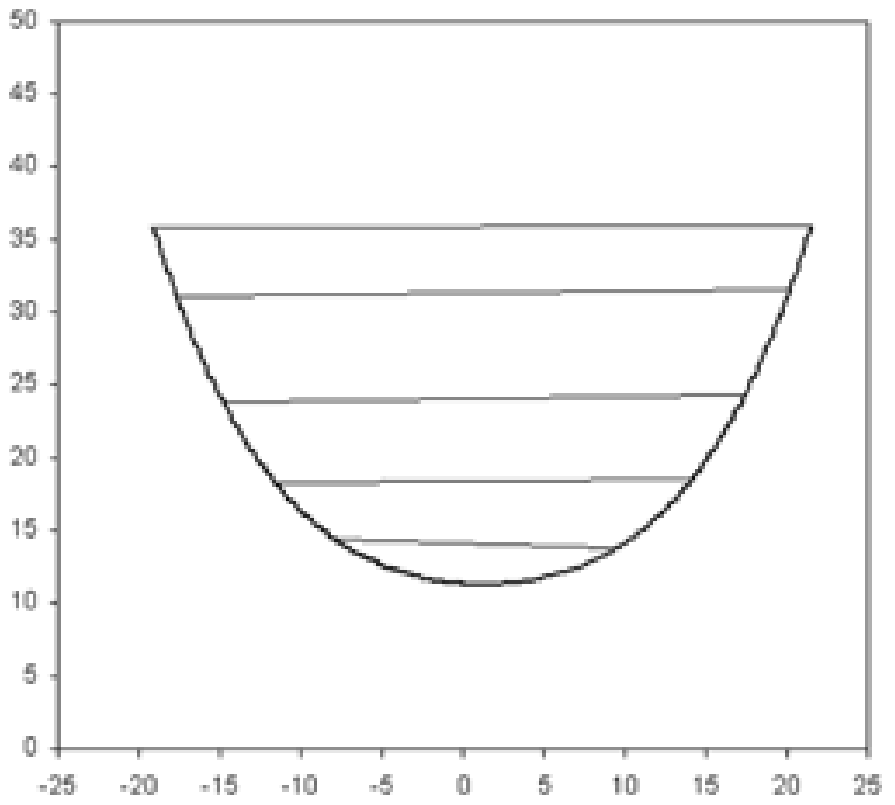

Fig. 2. Arch shape described by an orthogonal fourth order polynomial, with antimeric points joined to illustrate the degree of asymmetry.

where $\varphi_{1}, \varphi_{2}, \varphi_{3}$, and $\varphi_{4}$ are orthogonal polynomials and their coefficients are the last unprimed coefficients of each of the four equations respectively. However, this is only true in the case of equally-spaced data, a fact overlooked in the original paper. Even were it appropriate for use on unequally-spaced data, the subsequent partition of variance that was presented (cited from Ostle, 1958) was also incorrect, a fact which can be easily verified by application to the equally-spaced data from the same paper.

Kendall (1959) presented the analysis of equallyspaced $x$-values as a special case of the more general usage of orthogonal polynomials for all data-types. Robson (1959) extended the analysis of non-equally spaced $x$-values by presenting a simple recursive procedure to estimate appropriate orthogonal polynomial equations. An alternative construction procedure, also recursive but requiring the solution of $r$ linear equations for the construction of $\int_{r}\left(x_{i}\right)$ was described by Grandage (1958). Robson's (1959) methodology is robust and efficient and remains the method of choice for both equally- and unequally-spaced arch data. For the full methodology, a detailed examination of the original paper is recommended.

A simplified protocol appropriate for fitting a fourth-

TABLE 4. $X$ and $Y$ values for the dental arch used in the illustrative example

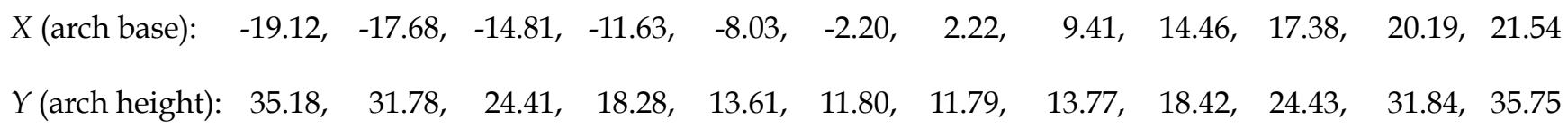


TABLE 3. Partition of variation

\begin{tabular}{lcrl}
\hline \multicolumn{1}{c}{ Source } & d.f. & Sum of Squares & \\
\hline Total11 & 935.87 & & \\
Symmetry $\left(b_{2}+b_{4}\right)$ & 2 & 932.19 & \\
$\quad$ Quadratic $\left(b_{2}\right)$ & 1 & 918.21 & $\ldots V_{2}$ \\
$\quad$ Quartic $\left(b_{4}\right)$ & 1 & 13.98 & $\ldots V_{4}$ \\
Asymmetry $\left(b_{1}+b_{3}\right)$ & 2 & 1.00 & \\
$\quad$ Linear $\left(b_{1}\right)$ & 1 & 1.00 & $\ldots V_{1}$ \\
$\quad$ Cubic $\left(b_{3}\right)$ & 1 & 0.00 & $\ldots V_{3}$ \\
Residual & 7 & 2.68 & \\
\hline
\end{tabular}

order orthogonal polynomial to arch shape data, and the subsequent partition of variance (Table 3 ) and derivation of shape-indices is presented below. The worked example uses data from a single arch (Hughes et al., 2001) for illustrative purposes (Fig. 1). The data are listed in Table 4 .

$$
\begin{aligned}
& \xi_{0}=\frac{1}{\sqrt{\mathrm{n}}} \\
& \xi_{1}=\frac{x-\xi_{0} \sum x \xi_{0}}{\sqrt{\sum\left(x-\xi_{0} \sum x \xi_{0}\right)^{2}}}
\end{aligned}
$$$$
\xi_{2}=\frac{\mathrm{x}-\xi_{0} \sum x^{2} \xi_{0}-\xi_{1} \sum x^{2} \xi_{1}}{\sqrt{\sum\left(x-\xi_{0} \sum x^{2} \xi_{0}-\xi_{1} \sum x^{2} \xi_{1}\right)^{2}}}
$$$$
\xi_{3}=\frac{x-\xi_{0} \sum x^{3} \xi_{0}-\xi_{1} \sum x^{3} \xi_{1}-\xi_{2} \sum x^{3} \xi_{2}}{\sqrt{\sum\left(x-\xi_{0} \sum x^{3} \xi_{0}-\xi_{1} \sum x^{3} \xi_{1}-\xi_{2} \sum x^{3} \xi_{2}\right)^{2}}}
$$$$
\xi_{4}=\frac{x-\xi_{0} \sum x^{4} \xi_{0}-\xi_{1} \sum x^{4} \xi_{1}-\xi_{2} \sum x^{4} \xi_{2}-\xi_{3} \sum x^{4} \xi_{4}}{\sqrt{\sum\left(x-\xi_{0} \sum x^{4} \xi_{0}-\xi_{1} \sum x^{4} \xi_{1}-\xi_{2} \sum x^{4} \xi_{2}-\xi_{3} \sum x^{4} \xi_{4}\right)^{2}}}
$$

$$
\begin{array}{ll}
\mathrm{b}_{1}=\sum \mathrm{y} \xi_{1}=1.00 & \mathrm{SS}_{1}=\mathrm{b}_{1}{ }^{2} \\
\mathrm{~b}_{2}=\sum \mathrm{y} \xi_{2}=30.30 & \mathrm{SS}_{2}=\mathrm{b}_{1}{ }^{2} \\
\mathrm{~b}_{3}=\sum \mathrm{y} \xi_{3}=0.04 & \mathrm{SS}_{3}=\mathrm{b}_{3}{ }^{2} \\
\mathrm{~b}_{4}=\sum \mathrm{y} \xi_{4}=3.74 & \mathrm{SS}_{4}=\mathrm{b}_{4}{ }^{2}
\end{array}
$$

Total SS $=\frac{\sum \mathrm{y}^{2}-\left(\sum \mathrm{y}\right)^{2}}{\mathrm{n}}=935.87$

Total SS explainable by regression:

$$
\mathrm{R}^{2}=\frac{935.87-2.68}{935.87}=1.00
$$

Shape indices can be calculated as outlined earlier. Total symmetry $=99.89 \%$ is composed of taperedness $(98.50 \%)$ plus squaredness $(1.5 \%)$. Total asymmetry $=0.11 \%$ is composed of lopsidedness $(100.00 \%)$ plus tiltedness $(0.00 \%)$. The relative magnitudes of these indices are illustrated in Figure 2, which shows the fitted curve with connected antimeres.

\section{CONCLUSION}

Lu's original 1966 paper remains of value for illustrating the utility of orthogonal polynomials in the analysis of arch shape data, and clearly the original theoretical considerations were of merit. Unfortunately, the numerous mathematical errors contained within the paper make its application to real-world data misleading and inaccurate. The corrections outlined in the present paper should now enable researchers to carry out more accurate and reliable 
quantitative assessments of dental arch form using the orthogonal polynomial approach.

\section{ACKNOWLEDGEMENTS}

This study was supported by a grant from the National Health and Medical Research Council (NH \& MRC) of Australia.

\section{LITERATURE CITED}

Battagel JM. 1996. Individualized catenary curves: their relationship to arch form and perimeter. Br J Orthod 23:21-28.

Fisher RA. 1921. Studies in crop variation. I. An examination of the yield of dressed grain from Broadbalk. J Agric Sci 11:107.

Fisher RA, Yates F. 1957. Statistical tables for biological, agricultural and medical research. New York: Hafner.

Grandage A. 1958. Orthogonal coefficients for unequal intervals. Biometrics 14:287-289.

Hrdlička A. 1916. Contribution to the anthropology of Central and Smith Sound Eskimos. Ann Pap Am Nat Hist 5:177-285.

Hughes TE, Richards LC, Townsend GC. 2001. Dental arch forms in young Australian twins. In: Brook A, editor. Dental Morphology 2001. Sheffield: Sheffield Academic Press Ltd, p 309-320.

Jones ML, Richmond S. 1989. An assesment of the fit of a parabolic curve to pre- and post-treatment dental arches. Br J Orthod 16:85-93.

Kasai K, Richards LC, Townsend GC, Kanazawa E, Tadamasa I. 1995. Fourier analysis of dental arch form in South Australian twins. Anthropol Sci 103:39-48.

Kendall MG. 1959. The advanced theory of statistics, 3rd ed. Vol. II. New York: Hafner.

$\mathrm{Lu} \mathrm{KH.} \mathrm{1966.} \mathrm{An} \mathrm{orthogonal} \mathrm{analysis} \mathrm{of} \mathrm{the} \mathrm{form,}$ symmetry and asymmetry of the dental arch. Arch Oral Biol 11:1057-1069.

Moorrees CFA. 1959. The dentition of the growing child. Cambridge: Harvard University Press.

Ostle B. 1958. Statistics in research. Iowa: Iowa State University Press.

Richards LC, Townsend GC, Brown T, Burgess VB. 1990. Dental arch morphology in South Australian twins. Arch Oral Biol 35:983-989.

Robson DS. 1959. A simple method for constructing orthogonal polynomials when the independent variable is unequally spaced. Biometrics 15:187-191.

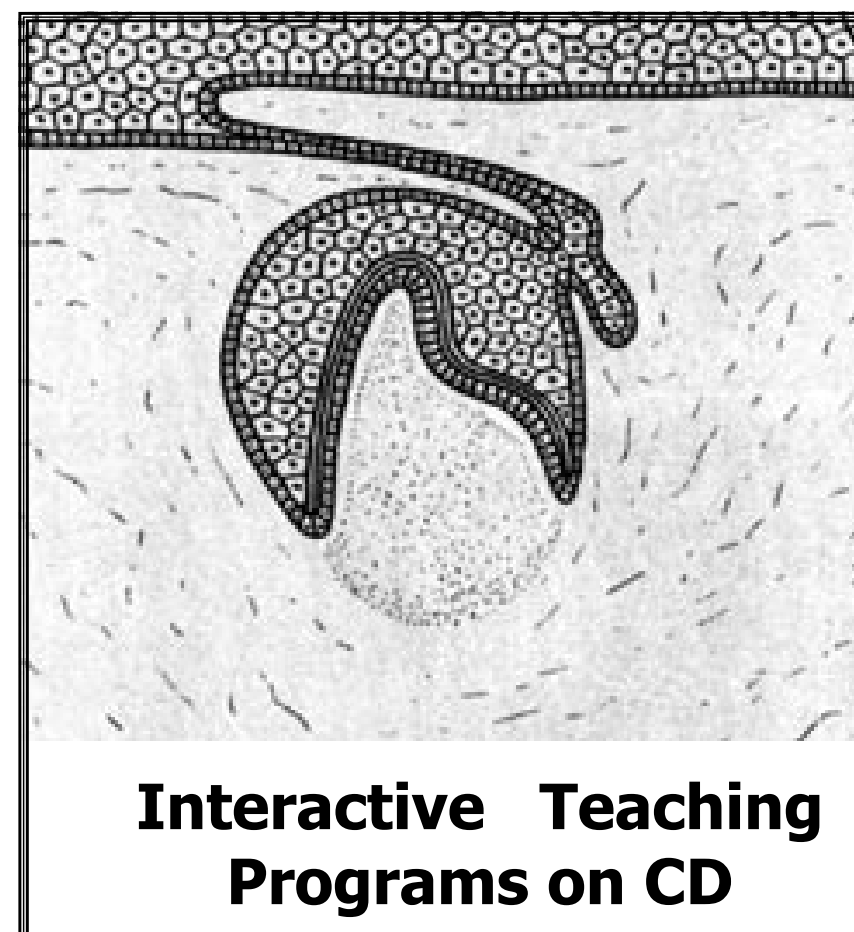

Two interactive programs on CD: Development of the Tooth Germ covers development of the tooth from initiation to formation of the root, not including development of the specific dental tissues. Navigation is via a simple menu structure with 10 chronological stages copiously illustrated with diagrams, clinical photographs and histological material.

Development of the Face, Palate and Tongue considerččs aspects of normal development of the neural crest, pharyngeal apparatus, face, palate and tongue and consequences of abnormal development, with a wide range of clinical examples. As well as providing many static images and animated diagrams, morphing techniques have been applied to scanning electron micrographs to provide movie sequences showing the structures actually changing.

Both programs include a quiz section. They run under Windows 3.11 or later. A 486 IBM compatible PC with $8 \mathrm{Mb}$ RAM and approximately 20 $\mathrm{Mb}$ free hard disk space is required.

How to order: The price of these CDs is US $\$ 40$ each for an individual copy or US $\$ 180$ each for a site licence. Cheques should be made payable to GGHB Endowment Fund 40-42 and sent to:

Dr. Marie E. Watt

Glasgow Dental School

378 Sauchiehall Street

Glasgow G2 3JZ U.K. 